\title{
Optical millimeter wave signal generation with frequency 16-tupling using cascaded MZMs and no optical filtering for radio over fiber system
}

\author{
M. Baskaran ${ }^{*^{*}}$ and R. Prabakaran ${ }^{2^{*}}$
}

\begin{abstract}
Background: An optical Millimeter Wave (MM-Wave) generation with high frequency multiplication factor is very attractive solution to avoid the use of high frequency local oscillators at the central station.

Methods: In this paper, a frequency 16 tupling technique is demonstrated with the use of four cascaded MZMs. With the proper adjustment of DC bias of the Mach-Zehnder Modulators (MZM), amplitude and phases of the RF Local oscillators, MM-Wave with a frequency 16 times the input RF LO is demonstrated.

Results: The cascaded structure has resulted very low modulation index of 2.827 and $54 \mathrm{~dB}$ and $42 \mathrm{~dB}$ of OSSR and RFSSR respectively. Analysis of both optical and RF sided band suppression ratios under non-ideal cases are also simulated and presented. Further, 10 Gbps NRZ data transmission performance over 50 km Single Mode Fiber (SMF) is also evaluated.
\end{abstract}

Conclusions: The proposed structure can tolerate up to few degrees of non-ideal parameter variation and is very simple to realize.

Keywords: Optical millimeter-wave, Mach-Zehnder Modulator, Radio over Fiber, Laser Diode

\section{Background}

In the recent years, there is a growing demand for the high speed data rate services, but which cannot be supported by existing congested frequency spectrum. To overcome the spectral congestion and support future traffic demands, use of MM-Wave frequency band has been proposed. The MM-Wave band ranging from 30 to $300 \mathrm{GHz}$ provides huge bandwidth (BW) of about $270 \mathrm{GHz}$, due to this abundant BW it can be used in future wireless communication and space communication to provide long-distance ultra-capacity services [1-3]. However, one of the major issues of the MM-Wave communication is the generation and transmission since electrical generation of such high frequency signal is very troublesome process. Hence optical MM-Wave generation and RoF transmission methods have been

\footnotetext{
* Correspondence: baski.maha@gmail.com; hiprabakaran@gmail.com 'Department of ECE, St. Joseph's Institute of Technology, Chennai -119, India ${ }^{2}$ BIT Campus, Anna University, Tiruchirappalli, Tamilnadu, India
}

proposed. Though there are several methods of optical MM-Wave generation techniques, external modulation based optical frequency multiplication techniques have gained much attention due to its higher modulation bandwidth, stability and flexibility [4-7]. Several optical frequency upconversion/multiplication techniques have been demonstrated with different frequency multiplication such as four, six, eight, ten and twelve [8-16]. Aforementioned techniques have utilized external modulation with optical carrier suppression (OCS) for the better unwanted sideband suppression using Lithium Niobate -Mach-Zehnder Modulators (LN-MZM). Similarly, such a OCS phenomenon can also be achieved using phase modulators (PM) with higher modulation index [17]. The OCS based frequency multiplication will greatly improve RoF transport systems due to its dispersion tolerance. However, MM-Wave generation with higher frequency multiplication factors play major role in limiting the need for high frequency electronic RF LOs at the Central Station. 


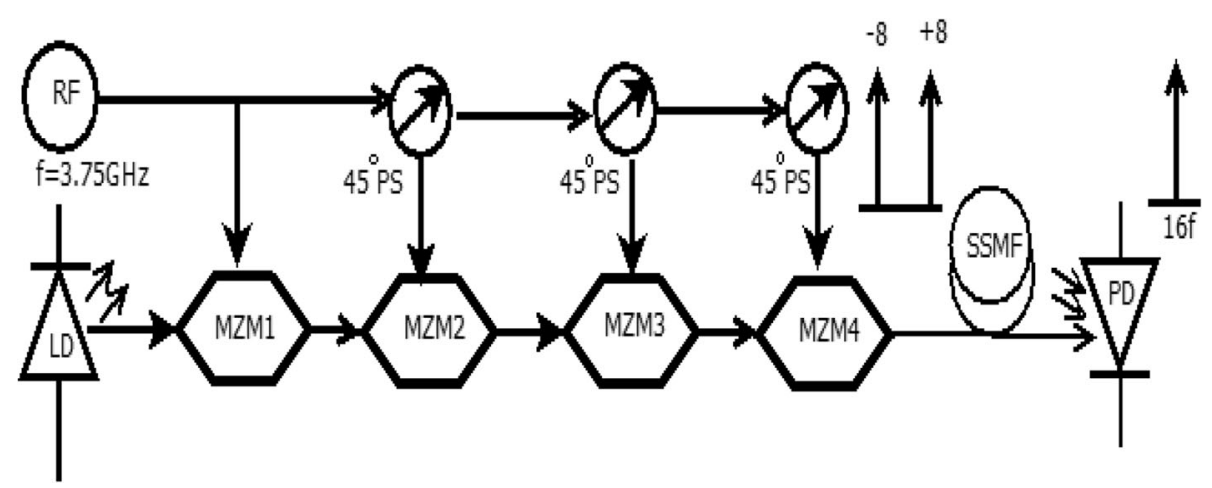

Fig. 1 Schematic diagram of frequency 16 tupling system using cascaded MZM. LD: laser diode; RF: Radio Fregency; MZM: Mach-Zehnder modulator; SSMF: standard single mode fiber; PS: Phase Shifter

But, single MZM cannot provide sufficient flexibility to select and generate the desired higher order optical sidebands and hence several series parallel configuration have been utilized to achieve high frequency multiplication factors. Hasan et al. have utilized array of four phase modulators to generate frequency octupling and 24 tupling [18]. Recently, several frequency 16- tupling techniques have been demonstrated using two cascaded MZMs with two tunable lasers [19], dual parallel MZMs [20], two cascaded dual parallel MZMs [21], and dual stage cascaded MZMs [22]. The modulation indices of these schemes are very high and used complex circuit arrangements. The higher modulation indices will have low conversion efficiency. Moreover, RFSSR and OSSRs of the proposals [19-21] are low. Hence in this paper, a cascaded combination of four MZMs is utilized to reduce the modulation index and improve the optical and RF sideband suppression ratios.
The organization of this paper is as follows, section 2 describes the mathematical modeling of the proposed technique section 3 demonstrates the numerical simulation results and finally section 4 concludes.

\section{Methods}

The schematic diagram of the generation of $60 \mathrm{GHz}$ milli-meter wave is shown in Fig. 1, which predominantly comprises a laser diode (LD), Mach-Zehnder Modulator (MZM), Standard single mode fiber (SSMF) and photodetector (PD). The RF LO can be specified as

$$
V_{r f}(t)=V_{s} \cos \left(\omega_{R F} t\right)
$$

$\omega_{R F}$ being the angular frequency and $V_{S}$ the amplitude of the microwave source.

A continuous wave (CW) Laser centered at $193.1 \mathrm{THz}$ can be expressed mathematically [23] as

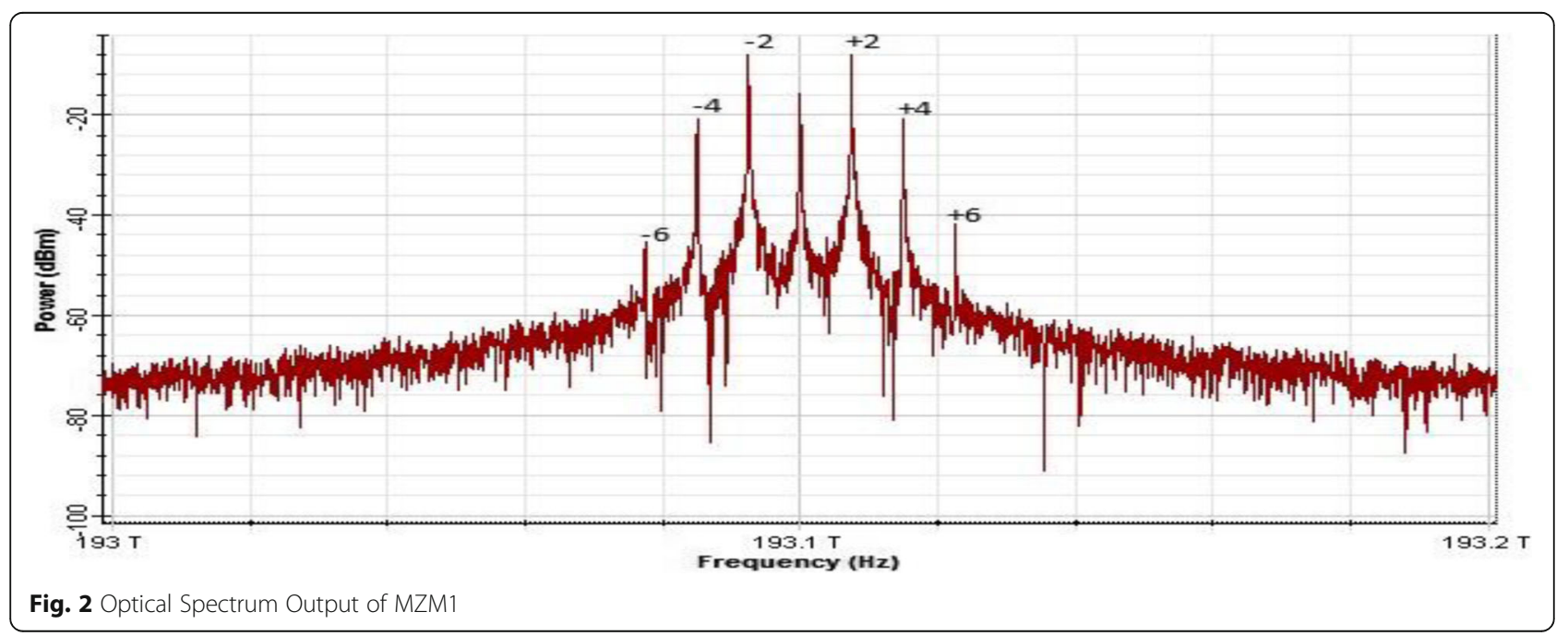




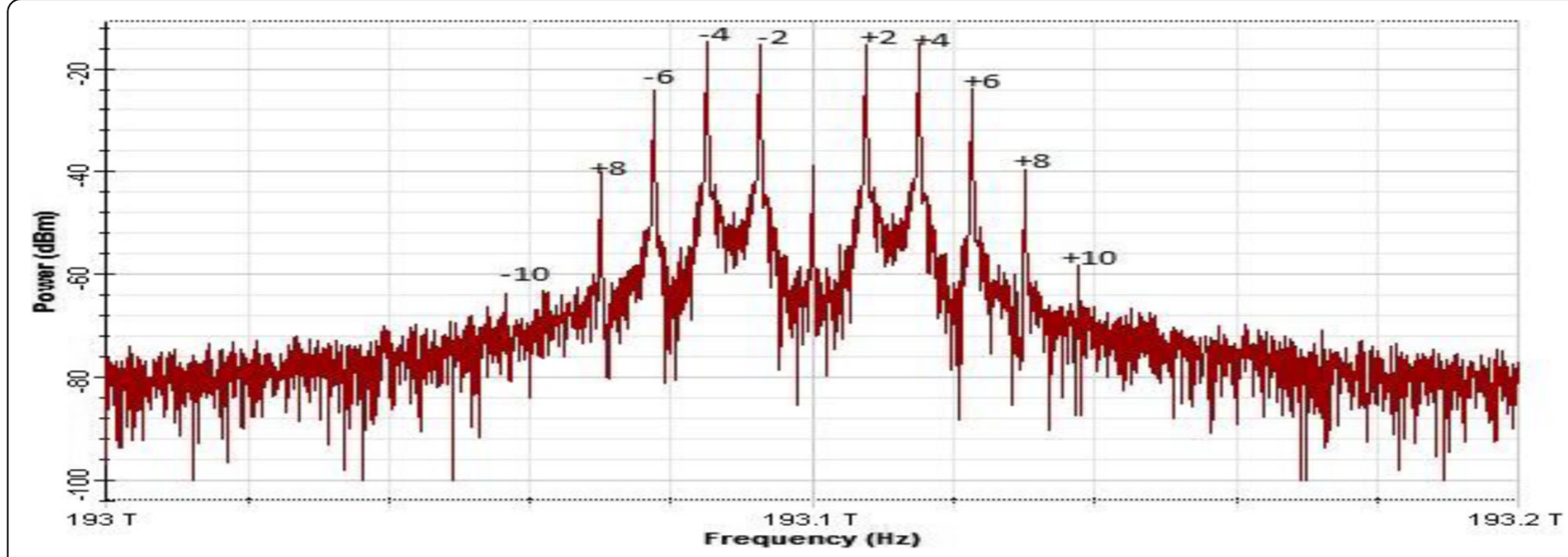

Fig. 3 Optical Spectrum Output of MZM2

$$
E_{i n}(t)=E_{c} e^{j \omega_{c} t}
$$

where $E_{c}$ and $\omega_{C}$ are the amplitude and angular frequency of the optical carrier signal and the output of the $\mathrm{MZM}_{1}$ is given by using Bessel function

$$
E_{m z m 1}(t)=\frac{\alpha E_{c} e^{j \omega_{c} t}}{2}\left[e^{-j m \sin \left(\omega_{R F} t\right)} e^{-j \pi / 2}+e^{j m \sin \left(\omega_{R F} t\right)} e^{j \pi / 2}\right]
$$

where $\mathrm{m}=\frac{\pi V_{S}}{V_{\pi}}$ is the modulation index, $\alpha$ is the insertion loss of the MZM, eq. 3 can be simplified using Jacobi-Anger expansion and the output can be written [24] as

$$
\begin{gathered}
\left.E_{m z m 1}(t)=\alpha E_{c}\left[\sum_{n=-\infty}^{n=\infty} J n(m) e^{j\left[\left(\omega_{c}+n \omega_{R F}\right) t+\frac{n \pi}{2}\right.}\right] \cos \left(\frac{n \pi}{2}\right)\right] . \\
E_{m z m 1}(t)=\alpha E_{c} e^{j \omega_{c} t}\left[J_{0}(m)+J_{2}(m)\left[e^{j 2 \omega_{r f} t}+e^{-j 2 \omega_{r f} t}\right]\right. \\
+J_{4}(m)\left[e^{j 4 \omega_{r f} t}+e^{-j 4 \omega_{r f} t}\right]+J_{6}(m)\left[e^{j \omega \omega_{r f} t}+e^{-j 6 \omega_{r f} t}\right]
\end{gathered}
$$

Figure 2 suggests the presence of only even order sidebands. Eq. 4 accords well with the obtained optical spectrum after MZM1.

This output is fed to MZM 2 in which an electrical phase shift of $45^{\circ}$ is introduced. At this stage the output can be expanded as

$$
E_{m z m 2}(t)=\frac{\alpha^{2}}{2} E_{c} \sum_{n=-\infty}^{n=\infty} J_{n}^{2}(m) e^{j\left[\left(\omega_{c}+2 n \omega_{R F}\right) t+n \pi\right]} \cos ^{2}\left(\frac{n \pi}{2}\right) e^{j n \pi / 4}
$$

$$
\begin{aligned}
E_{m z m 2}(t) & =\alpha^{2} E_{c} e^{j \omega_{c} t}\left\{J_{0}^{2}(m)+\left[e^{j 2 \omega_{r f} t}+e^{-j 2 \omega_{r f} t}\right]\right. \\
& {\left[-J_{0}(m) J_{2}(m)(1+j)+J_{2}(m) J_{4}(m)(1-j)\right.} \\
& \left.+J_{6}(m) J_{4}(m)(1+j)\right] \\
& +\left[e^{j 4 \omega_{r f} t}+e^{-j 4 \omega_{r f} t}\right]\left[j J_{2}^{2}(m)\right] . \\
& +\left[e^{j 6 \omega_{r f} t}+e^{-j 6 \omega_{r f} t}\right]\left[-J_{0}(m) J_{6}(m)(1-j)\right. \\
& \left.+J_{2}(m) J_{4}(m)(1-j)\right]+\left[e^{j 6 \omega_{r f} t}+e^{-j 8 \omega_{r f} t}\right] \\
& {\left.\left[-J_{4}^{2}(m)\right]+\left[e^{j 10 \omega_{r f} t}+e^{-j 10 \omega_{r f} t}\right]\left[J_{4}(m) J_{6}(m)\right]\right\} }
\end{aligned}
$$

Output of MZM2 is shown in Fig. 3 which indicates the presence of 2nd, 4th, 6th, 8th and 10th order optical sidebands which accords well with the eq. 6 .

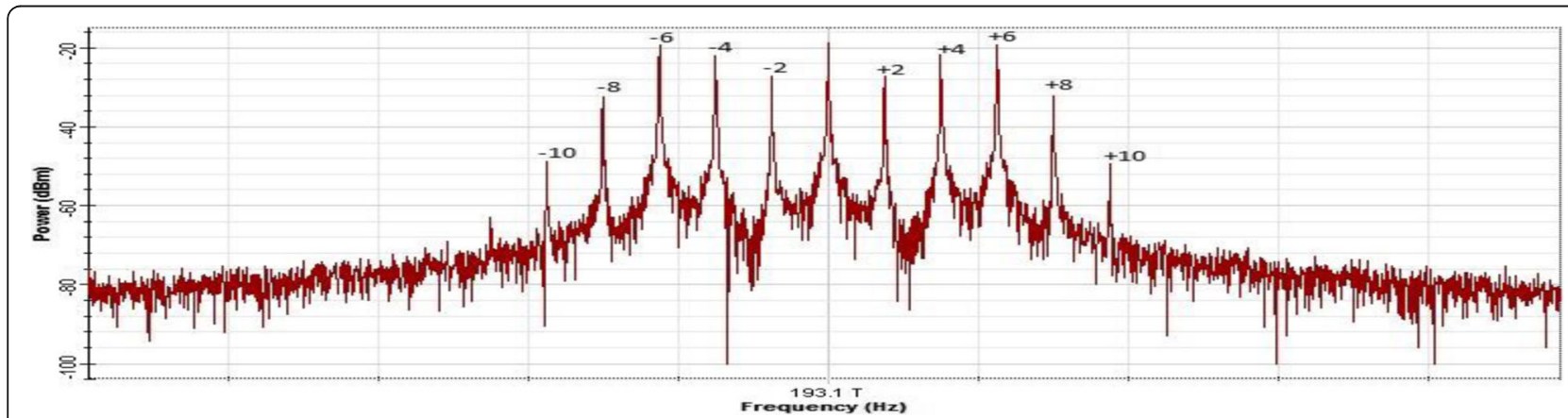

Fig. 4 Optical Spectrum Output of MZM3 
Table 1 Simulation parameters

\begin{tabular}{ll}
\hline Parameter & Values \\
\hline Bit Rate & $10 \mathrm{Gbps}$ \\
Time window & $1.28 \mathrm{e}-0.08 \mathrm{~s}$ \\
Sample rate & $640 \mathrm{GHz}$ \\
Sequence length & $128 \mathrm{Bits}$ \\
Samples per Bit & 64 \\
Number of samples & 8192 \\
Sensitivity & $-100 \mathrm{dBm}$ \\
Resolution & $0.01 \mathrm{~nm}$ \\
Fiber length & $50 \mathrm{Km}$ \\
Optical power of CW laser & $2 \mathrm{dBm}$ \\
Linewidth of CW laser & $10 \mathrm{MHz}$ \\
Wavelength of CW laser & $1550 \mathrm{~nm}$ \\
Dispersion of optical fiber & $16.75 \mathrm{ps} / \mathrm{nm} / \mathrm{km}$ \\
Switching Bias Voltage of MZM & $4 \mathrm{~V}$ \\
Switching RF Voltage of MZM & $4 \mathrm{~V}$ \\
Photodiode responsivity & $0.8 \mathrm{~A} / \mathrm{W}$ \\
Extinction Ratio of MZ-5 & $30 \mathrm{~dB}$ \\
\hline
\end{tabular}

Output obtained after MZM2 serves as an input to the next stage. An electrical phase shift of $45^{\circ}$ is again introduced at this stage. The spectrum obtained after MZM3 is shown in Fig. 4.

$$
\begin{aligned}
E_{m z m 3}(t)= & \alpha E_{m z m 2}(t)\left\{J_{0}(m)-J_{2}(m)\left[e^{j 2 \omega_{r f} t}+e^{-j 2 \omega_{r f} t}\right]\right. \\
& \left.+J_{4}(m)\left[e^{j 4 \omega_{r f} t}+e^{-j 4 \omega_{r f} t}\right]-J_{6}(m)\left[e^{j 6 \omega_{r f} t}+e^{-j 6 \omega_{r f} t}\right]\right\}
\end{aligned}
$$

The spectrum obtained after MZM3 is shown in Fig. 4. which shows a good agreement with equation (7). The output of MZM3 is fed to MZM4 which is driven by an RF LO with $45^{\circ}$ phase shift. From the expression obtained at the 4th stage it can be readily inferred that only 8th order sidebands exist and the rest are suppressed. According to the below eq. (8), the zeroth-order and fourth-order Bessel function become zero due to the modulation index and the electrical phase shift of $135^{\circ}$ [20]. Due to their extremely low power, the higher order optical sidebands greater than eighth-order can be neglected. Now the eq. 8 can be approximately written as,

$$
E_{m z m 4}(t) \approx\left(\alpha^{4} / 2\right) J_{2}^{4}(m) E_{c} e^{\left(j \omega_{c} t\right)}\left[e^{\left(j 8 \omega_{r f} t\right)+\left(-j 8 \omega_{r f} t\right)}\right]
$$

At the receiving end, the photo current is given [25] by,

$$
\mathrm{I}(t)=\mu|E(t)|^{2}
$$

where $\mu$ is the responsivity of the photo detector. The eq. (9) can be simplified neglecting the all other terms as,

$$
\mathrm{I}(t)=\frac{\mu \alpha^{2}|E(t)|^{2}}{4} \cos \left(16 \omega_{R F} t\right)
$$

The MM-Wave generation employs the frequency 16 tupling scheme using cascaded Mach Zehnder modulator. The source is a continuous wave laser which emits a light signal that serves as a carrier to drive the MZM. The usage of MZM is due to the advantages it offers in terms of modulation bandwidth, driving voltage and chirp. MZM requires a very low driving voltage but in turn offers a high modulation bandwidth. The MZM here, is employed for optical harmonic generation for optical frequency multiplication. The purpose of choosing MZM over other external modulators is because MZM has a predictable transfer function shape. An electrical phase shift of 45 degrees is introduced between them, the accurate adjustment of which aids in the suppression of all the harmonics other than the ones

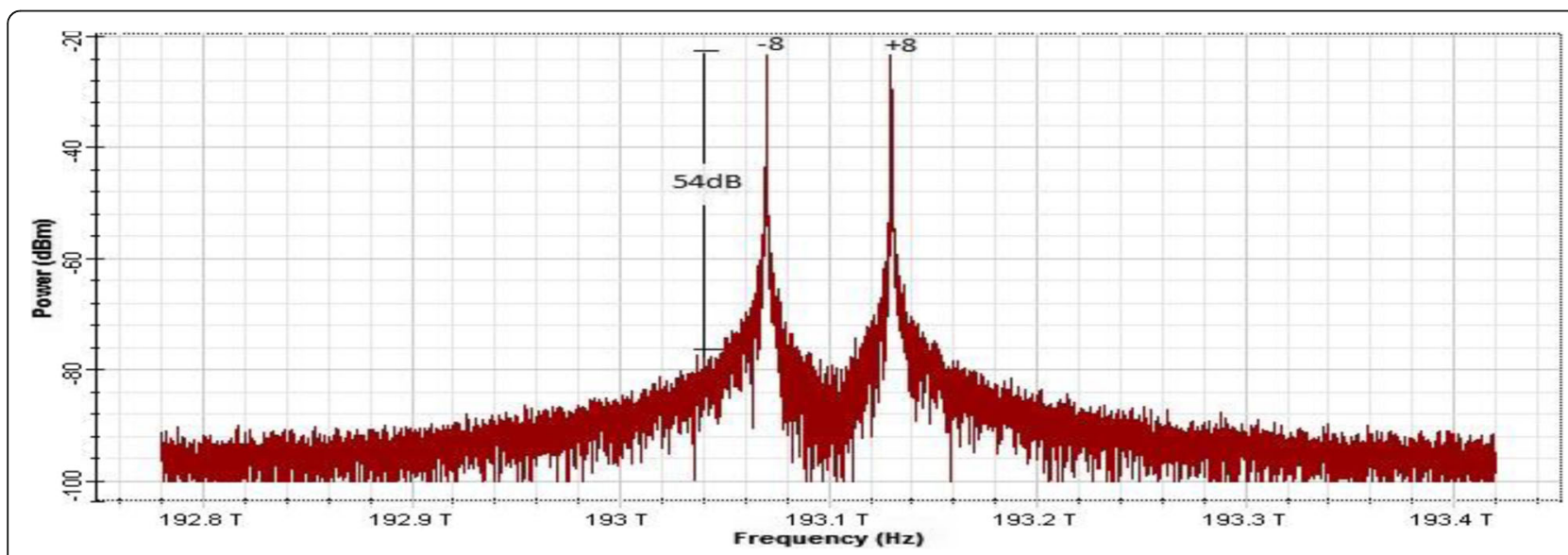

Fig. 5 Optical Spectrum Output of MZM4 


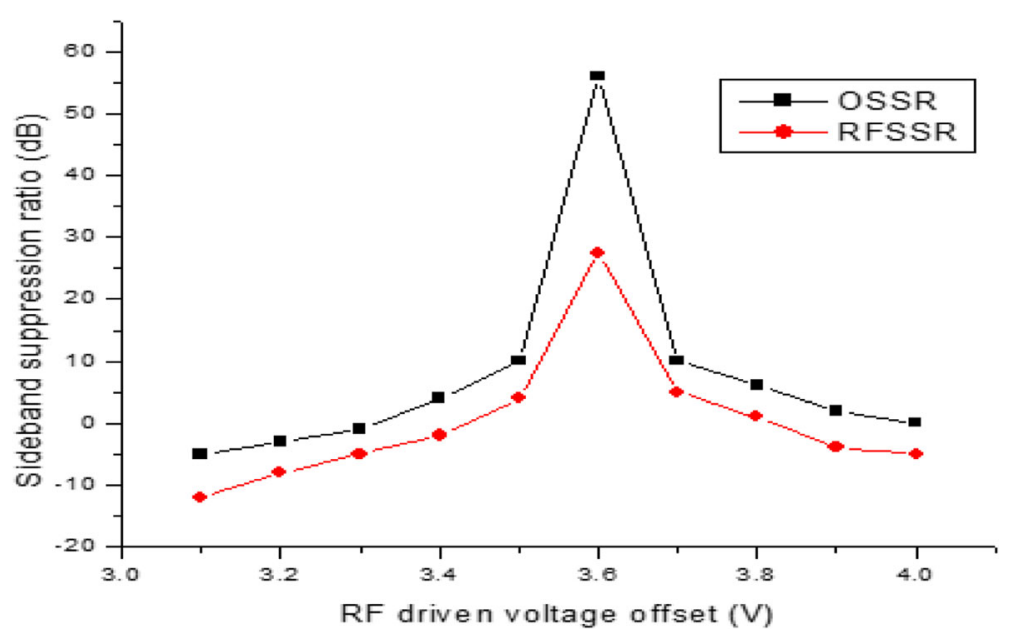

Fig. 6 Influence of RF voltage variation

desired. It is important to note that the MZMs operate at maximum transmission point. The first MZM suppresses all the odd harmonics present in the spectrum, leaving behind only the even harmonics in the spectrum. Before the first stage output is fed to the second MZM, the RF signal driving MZM2 is phase shifted by 45 degrees. The phase shifted RF signal is fed as the input to the second and the third MZMs, which further suppress the even harmonics, leaving behind the 8th harmonic on each side. Proper and efficient adjustment of dc biasing ensures that all the harmonics are suppressed except for the 8th harmonic on either side. Upon the photo detection, peak power is achieved in the 16th harmonics, while all the other harmonics are eliminated. The frequency of the input RF LO signal is $3.75 \mathrm{GHz}$, which generates an output signal with a frequency of $60 \mathrm{GHz}$ which is 16 times the input RF LO. By cascading four MZMs with the output of each being successively phase-shifted by 45 degrees, we obtain peak power only in the 8th harmonic on either side, while completely eliminating and suppressing the other harmonics including the carrier, the presence of which is undesirable. The suppression of the carrier is mainly to avoid the wastage of power in transmitting the carrier because it is only the 8 th harmonics that are desired to avoid compromise in the performance of the transmission. This $60 \mathrm{GHz}$ frequency becomes important and extremely vital because it is the unlicensed band that supports a large number of applications involving high data rates.

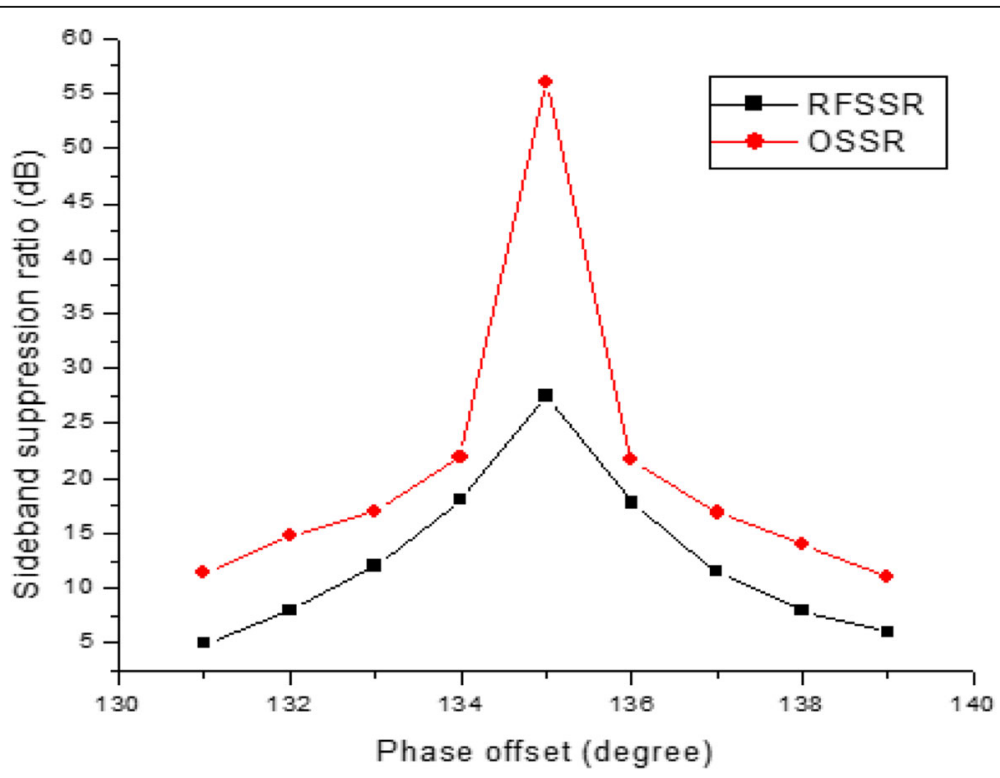

Fig. 7 Influence of phase difference 


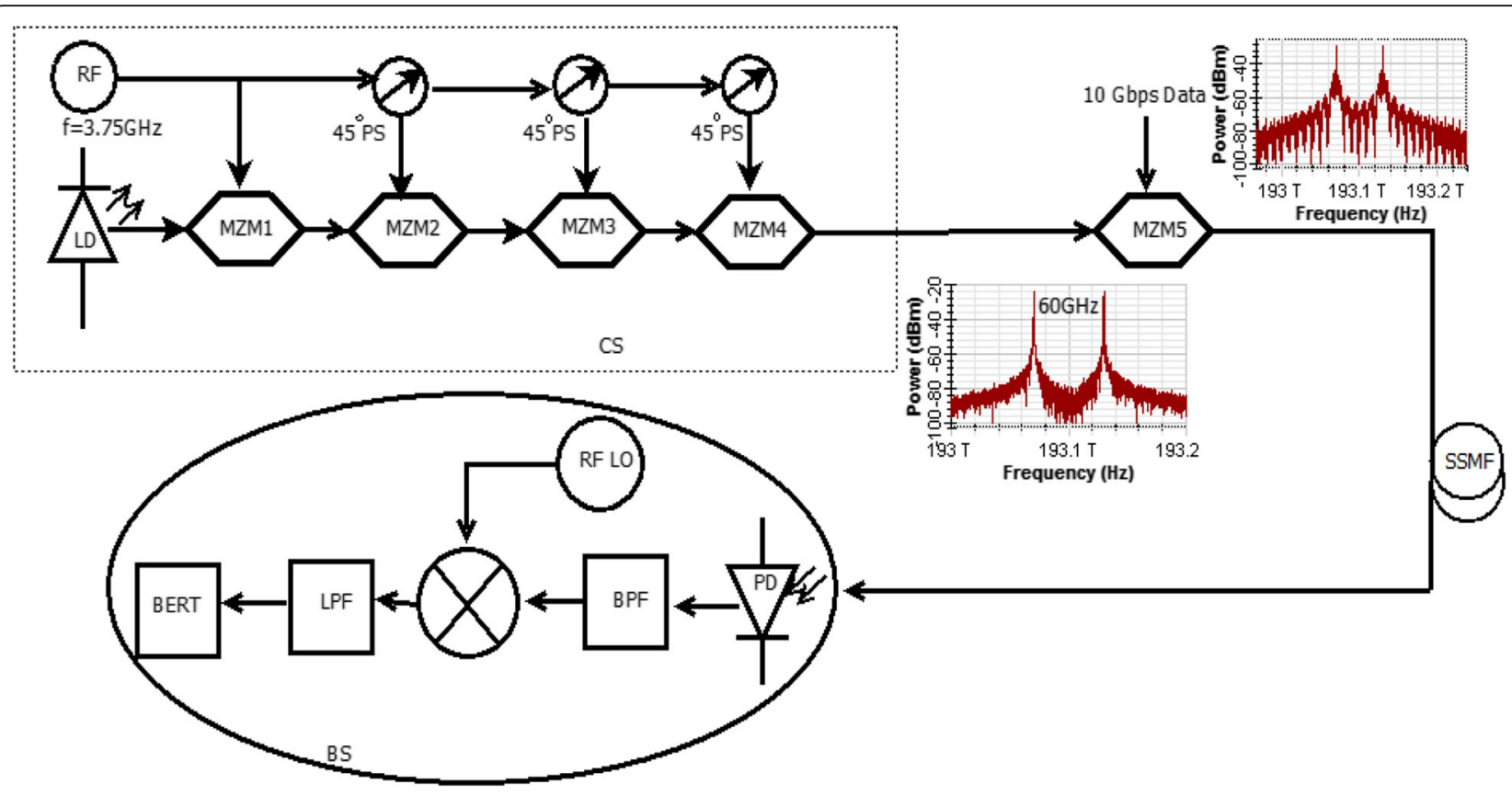

Fig. 8 Simulation setup of the transmission performance evaluation

\section{Simulation results and discussion}

Table 1 shows the different parameters and the specifications to be set to obtain the required output. We have used a $50 \mathrm{~km}$ long optical fiber cable with an attenuation of $0.2 \mathrm{~dB}$ per $\mathrm{km}$ which is ideal. The exact specifications and the values of the different parameters used are listed as follows

The schematic used for evaluating the proposed technique is shown in Fig. 1. A concept-proof RoF link is built based on the OptiSystem platform. A continuous wave laser source with $193.1 \mathrm{THz}$ (equivalent wavelength of $1552.52 \mathrm{~nm}$ ) central frequency is launched in to the MZM1. The attenuation at $1550 \mathrm{~nm}$ (third window) wavelength is minimum compared with $1300 \mathrm{~nm}$ (second window) or $850 \mathrm{~nm}$ (first window) in optical communication systems. This window has intrinsically lowest loss of about $0.2 \mathrm{~dB} / \mathrm{km}$. The MZM1 is biased at the maximum transmission point (MTP) with a switching bias voltage of $4 \mathrm{~V}$

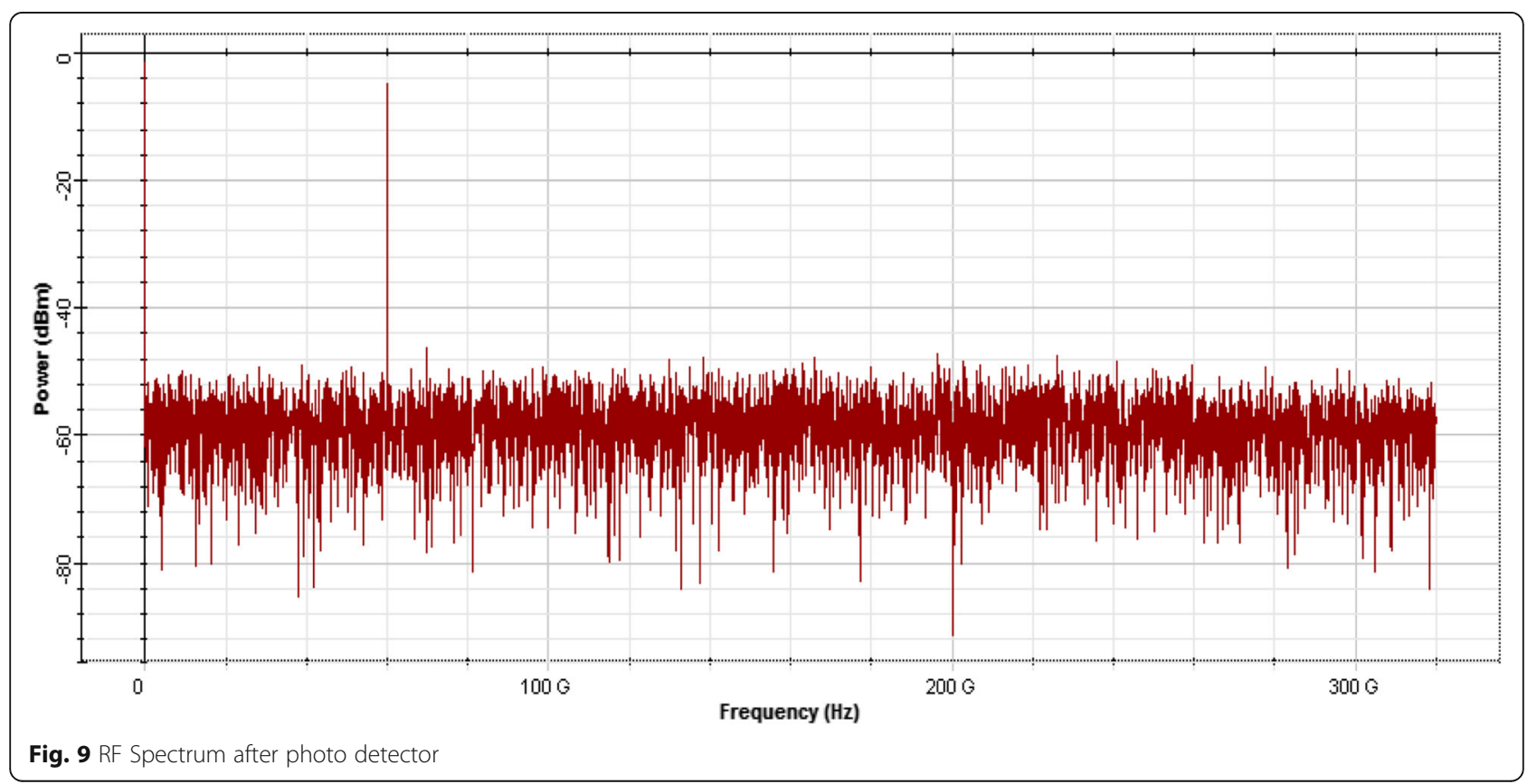




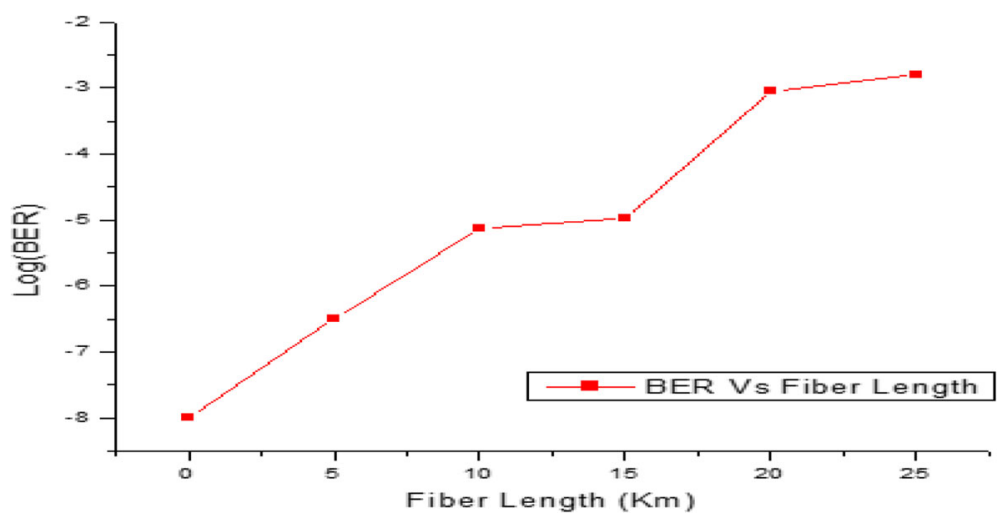

Fig. 10 Bit Error Rate Vs. Length of the fiber

and driven by $3.75 \mathrm{GHz}$. RF LO with amplitude of $3.6 \mathrm{~V}$. The extinction ratio is set to infinite. Due to MTP, the output of the MZM1 consists only the even order sidebands as shown in Fig. 2. Output of the first MZM is then passed on to the MZM2 to MZM4 which are driven by RF LO with 45 degrees phase shift. The MZMs are biased at the MTP; hence the output contains only 8 th order sidebands. The modulation index is fixed is set to 2.827, so that the output power of the 8th order sideband is $-21.7557 \mathrm{dBm}$ at MZM4 as shown in Fig. 5.

The eight order sideband appears at $193.07 \mathrm{THz}$ and 193.13 THz. The output signal shown in the Fig. 5. The difference between the generated eight order sidebands is observed to be $60 \mathrm{GHz}$ and is transported over $50 \mathrm{~km}$ single mode fiber with attenuation co-efficient of $0.2 \mathrm{~dB} /$ $\mathrm{km}$ and the dispersion of $16.75 \mathrm{ps} / \mathrm{nm}-\mathrm{km}$. An optical amplifier with sufficient gain is used to compensate the losses in the link. At the receiving side, a PIN photo diode receives and converts into electrical signal with $0.8 \mathrm{~A} / \mathrm{W}$ responsivity and $10 \mathrm{nA}$ dark current.

The effect of deviation of RF drive voltage from its ideal value is shown in Fig. 6. It is observable that OSSR and RFSSR remain unaffected when RF voltage drifts negligibly from the ideal value. As the value of RF voltage deviates from the ideal value, a corresponding increase in degradation of sideband suppression ratios is noticeable. Hence RF voltage deviation of less than $0.05 \mathrm{~V}$ is suggestive for an OSSR greater than $32 \mathrm{~dB}$ and RFSSR greater than $16.5 \mathrm{~dB}$.

Further, the effect of phase shift drifts between the microwave drive signals applied to MZMs from ideal values is shown in Fig. 7. For deviations less than $0.5^{\circ}$, the achievable OSSR and RFSSR are more than $30 \mathrm{~dB}$ and $22.5 \mathrm{~dB}$ respectively.

These optical sidebands undergo intensity modulation with 10 Gbps baseband signal which is depicted in Fig. 8. We make use of another MZM (MZ-5) for this purpose. As a consequence of signal modulation, optical sidebands are broadened, while modulation insertion loss attenuates the side bands to a negligible extent. The modulated signal is then photo-detected at the base station. Following this, filtering out of RF harmonics is achieved by means of a band pass filter (BPF) with a central frequency of $60 \mathrm{GHz}$.Subsequent stages in demodulation of $10 \mathrm{Gbps}$ signal include the employment of a $60 \mathrm{GHz}$ LO and a mixer followed by low pass filtering.

The received signal contains data modulated $60 \mathrm{GHz}$ MM-Wave signal. Figure 9 shows only dominant output of the RF spectrum. A $60 \mathrm{GHz}$ MM-Wave is generated from the $3.75 \mathrm{GHz}$ RF local oscillator. Simulations are repetitively performed considering varying fiber lengths and Bit Error Rate (BER) is computed. Figure 10 illustrates the plot of BER versus varying link distances; the performance of the generated $60 \mathrm{GHz}$ signal is acceptable.

\section{Conclusion}

In this paper, we have presented a novel 16 tupled MMWave generation technique using cascaded combination of four LN-MZMs and simultaneous RoF transmission system. The 8 th order sidebands are alone generated without any optical filters. Modulation index of the proposed scheme is very low compared to the prior arts. An error free transmission of $10 \mathrm{Gbps}$ data over $50 \mathrm{~km}$ SMF is demonstrated. The system can be practically implemented for the generation of optical MM-Waves and transmission of future multi-Gbps services to the customers. However, the bias drift issues of the proposed technique can be alleviated with the use of Polarization modulators.

\section{Abbreviations}

BER: Bit Error Rate; LD: Laser Diode; MM-Wave: Milli-Meter Wave; MTP: Maximum Transmission Point; MZM: Mach-Zehnder Modulator; OCS: Optical Carrier Suppression; RoF: Radio over Technology

\section{Acknowledgements}

The authors thankfully acknowledge Dr. K. Esakki Muthu, Assistant Professor of ECE, University VOC. College of Engineering, Thoothukudi-8. for his useful suggestions and discussions. 


\section{Authors contribution}

$\mathrm{MB}$ has worked out the mathematical derivations of the proposed system and carried out all the simulation works. RP has developed the concept and involved in the manuscript preparation. Both authors read and approved the final manuscript.

\section{Funding}

No funding agencies are involved in this research.

\section{Availability of data and materials}

All data and materials are freely available without any restriction.

\section{Competing interests}

The authors declare that they have no competing interest.

\section{Publisher's Note}

Springer Nature remains neutral with regard to jurisdictional claims in published maps and institutional affiliations.

Received: 2 February 2018 Accepted: 2 April 2018

Published online: 25 April 2018

\section{References}

1. Chen, S., Zhao, J.: The requirements, challenges, and technologies for $5 \mathrm{G}$ of terrestrial mobile telecommunication. IEEE Commun. Mag. 52(5), 36-43 (2014)

2. Li, X., Xiao, J., Yu, J.: Long-distance wireless mm-wave signal delivery at W-band. J Lightwave Technol. 34(2), 661-668 (2016)

3. $L i, X ., Y u, J ., X i a o, J .:$ Demonstration of ultra-capacity wireless signal delivery at W-band. J. Lightwave Technol. 34(1), 180-187 (2016)

4. Chow, C.W., Yeh, C.H., Sung, J.Y., Hsu, C.W.: Wired and wireless convergent extended-reach optical access network using direct-detection of all-optical OFDM super-channel signal. Opt. Express. 22(25), 30719-30724 (2014)

5. Huang, H.T., Shih, P.T., Lin, C.T., Cheng, Y.H., Liang, W.L., Ho, C.H., Wei, C.C., Ng'oma, A.: 2X2 MIMO OFDM-RoF system employing LMS-based equalizer with I/Q imbalance compensation at $60 \mathrm{GHz}$. IEEE Photonics Journal. 6(3), 1-7 (2014)

6. Shih, P.T., Chen, J., Lin, C.T., Jiang, W.J., Huang, H.S., Peng, P.C., Chi, S.: Optical millimeter-wave signal generation via frequency 12-tupling. J. Lightwave Technol. 28(1), 71-78 (2010)

7. Li, W., Wang, L.X., Zheng, J.Y., Li, M., Zhu, N.H.: Photonic MMW-UWB signal generation via DPMZM-based frequency up-conversion. IEEE Photon. Technol. Lett. 25(19), 1875-1878 (2013)

8. Lin, C.T., Shih, P.T., Chen, J., Xue, W.Q., Peng, P.C., Chi, S.: Optical millimeter wave signal generation using frequency quadrupling technique and no optical filtering. IEEE PhotonTechnol Lett. 20(12), 209-211 (2008)

9. Muthu, K.E. and Raja A.S. "Frequency quadrupling with improved OSSR and RFSSR for radio over fiber applications." Inventive Computation Technologies (ICICT), International Conference on. Vol. 2. Pp.1-4, IEEE, 2016

10. Gao, Y., Wen, A., Yu, Q., Li, N., Lin, G., Xiang, S., Shang, L.: Microwave generation with photonic frequency sextupling based on cascaded modulators. IEEE Photon. Technol. Lett. 2(12), 1199-1202 (2014)

11. Li, C., Mao, J., Dai, R., Zhou, X., Jiang, J.: Frequency-sextupling optoelectronic oscillator using a Mach-Zehnder interferometer and an FBG. IEEE Photon. Technol. Lett. 28(12), 1356-1359 (2016)

12. Lin, C.T., Shih, P.T., Jiang, W.J., Chen, J.J., Peng, P.C., Chi, S.: A continuously tunable and filterless optical millimeter-wave generation via frequency octupling. Opt. Express. 17(22), 19749-19756 (2009)

13. Hasan, M., Hall, T.J.: A photonic frequency octo-tupler with reduced RF drive power and extended spurious sideband suppression. Opt. Laser Technol. 81 115-121 (2016)

14. Muthu, K.E., Raja, A.S., Sevendran, S.: Optical generation of millimeter waves through frequency decupling using DP-MZM with RoF transmission. Opt. Quant. Electron. 49(2), 63 (2017)

15. Zhu, Z., Zhao, S., Zheng, W., Wang, W., Lin, B.: Filterless frequency 12-tupling optical millimeter-wave generation using two cascaded dual-parallel Mach-Zehnder modulators. Appl. Opt. 54(32), 9432-9440 (2015)

16. Muthu, K.E. and Raja, A.S: Improved filterless12-tupled optical MM-wave generation and $2.5 \mathrm{~Gb} / \mathrm{s}$ RoF transmission. Optoelectronics and advanced materials-rapid Communications, Vol. 10 No.11-12, pp.869-872, 2016

17. Lin, C.T., Chen, J.J., Dai, S.P., Peng, P.C., Chi, S.: Impact of nonlinear transfer function and imperfect splitting ratio of MZM on optical up-conversion employing double sideband with carrier suppression modulation. J. Lightwave Technol. 26(15), 2449-2459 (2008)

18. Hasan, M., Guemri, R., Maldonado-Basilio, R., Lucarz, F., de la Tocnaye, J.-L.d.B. , Hall, T.: Theoretical analysis and modeling of a photonic integrated circuit for frequency 8-tupled and 24-tupled millimeter wave signal generation, opt. Lett. 39, 6950-6953 (2014)

19. Chen, H., Ning, T., Jian, W., Pei, L., Li, J., You, H., Zhang, C.: D-band millimeterwave generator based on a frequency 16-tupling feed-forward modulation technique. Opt. Eng. 52(7), 076104 (2013)

20. Yin, X., Wen, A., Yang, C., Wang, T.: Studies in an optical millimeter-wave generation scheme via two parallel dual-parallel Mach-Zehnder modulators. J. Mod. Opt. 58(8), 665-673 (2011)

21. Zhu, Zihang, Shanghong Zhao, Xingchun Chu, and Yi Dong. "Optical generation of millimeter-wave signals via frequency 16-tupling without an optical filter." Optics Communications354 (2015): 40-47

22. Esakki Muthu, K., Sivanantha Raja, A., Shanmugapriya, G.: Frequency16-tupled optical millimeter wave generation using dual cascaded MZMs and 2.5 Gbps RoF transmission. Optik: Int. J. Light Electron Opt. 140, 338-346 (2017)

23. Chen, Y., Wen, A., Shang, L.: Analysis of an optical mm-wave generation scheme with frequency octupling using two cascaded Mach-Zehnder modulators. Opt. Commun. 283, 4933-4941 (2010)

24. Al-Sharee, N.A., Hassan, S.I.S., Malek, F., Ngah, R., Abbas, S.A., Aljunid, S.A.: A cost-effective method for high-quality $60 \mathrm{GHz}$ optical millimeterwave signal gener-ation based on frequency quadrupling. Prog. Electromagn. Res. 137, $255\{274(2013)$

25. Al-Shareefi, N.A., Hassan, S.I.S., Malek, F., Ngah, R., Aljunid, S.A., Fayadh, R.A., ALdhaibani, J.A., Rahim, H.A.: Development of a new approach for highquality quadrupling frequency optical millimeter-wave signal generation without optical filter. Prog. Electromagn. Res. 134, 189-208 (2013)

\section{Submit your manuscript to a SpringerOpen ${ }^{\circ}$ journal and benefit from:}

- Convenient online submission

- Rigorous peer review

Open access: articles freely available online

- High visibility within the field

Retaining the copyright to your article

Submit your next manuscript at $>$ springeropen.com 